\title{
QUASICONFORMAL MAPPINGS AND MINIMAL MARTIN BOUNDARY OF $p$-SHEETED UNLIMITED COVERING SURFACES OF THE COMPLEX PLANE
}

\author{
Hiroaki Masaoka and Shigeo Segawa \\ To the memory of Professor Nobuyuki Suita
}

\section{§1. Introduction}

Let $W$ be an open Riemann surface. We denote by $\Delta_{1}^{W}$ the minimal Martin boundary of $W$. In [L], it was showed that there exist open Riemann surfaces $F$ and $F^{\prime}$ quasiconformally equivalent to each other such that $F^{\prime}$ possesses nonconstant positive harmonic functions although $F$ does not possess nonconstant positive harmonic functions. This means that $\# \Delta_{1}^{F^{\prime}} \geq 2$ although $\# \Delta_{1}^{F}=1$, where $\# A$ stands for the cardinal number of a set $A$. Needless to say, the above $F$ and $F^{\prime}$ are of positive boundary, i.e. $F$ and $F^{\prime}$ admit the Green function (cf. [SN]). However, in case open Riemann surfaces $W$ and $W^{\prime}$ are of null boundary (i.e. not positive boundary), it does not seem to be known whether $\# \Delta_{1}^{W}=\# \Delta_{1}^{W^{\prime}}$ or not if $W$ and $W^{\prime}$ are quasiconformally equivalent to each other.

In this paper, we are concerned with $p$-sheeted unlimited covering surfaces of the complex plane C. Consider $p$-sheeted unlimited covering surfaces $R$ and $R^{\prime}$ of the complex plane $\mathbf{C}$ which are quasiconformally equivalent to each other. Then, it seems to be valid that $\# \Delta_{1}^{R}=\# \Delta_{1}^{R^{\prime}}$ (cf. [Sh], $[\mathrm{M}]$ ). The purpose of this paper is to give a partial answer to this conjecture. Namely,

MaIn TheORem. Let $R$ and $R^{\prime}$ be p-sheeted unlimited covering surfaces of $\mathbf{C}$ which are quasiconformally equivalent to each other. If $p=2$ or 3 , then it holds that $\# \Delta_{1}^{R}=\# \Delta_{1}^{R^{\prime}}$.

\section{§2. Preliminaries}

Hereafter we consider the punctured sphere $\hat{\mathbf{C}} \backslash\{0\}$ in place of the complex plane $\mathbf{C}$ since $\hat{\mathbf{C}} \backslash\{0\}$ is conformally equivalent to $\mathbf{C}$. Hence we assume that $R$ and $R^{\prime}$ in Main Theorem are $p$-sheeted unlimited covering surfaces of $\hat{\mathbf{C}} \backslash\{0\}$. Let $\Delta^{R}$ and $\Delta_{1}^{R}$ be as in $\S 1$, and $\pi$ the projection map from $R$ onto $\hat{\mathbf{C}} \backslash\{0\}$. Set $\mathbf{D}=\{z \in \mathbf{C}|| z \mid<1\}, \mathbf{D}_{0}=\mathbf{D} \backslash\{0\}$ and $R_{0}=\pi^{-1}\left(\mathbf{D}_{0}\right)$. It is well-known that $\Delta^{R_{0}}$ and $\Delta_{1}^{R_{0}}$ are identified with $\Delta^{R} \cup \pi^{-1}(\partial \mathbf{D})$ and $\Delta_{1}^{R} \cup \pi^{-1}(\partial \mathbf{D})$, respectively, where 
$\partial \mathbf{D}=\{|z|=1\}$. From now on we consider $\mathbf{D}_{0}$ (resp. $R_{0}$ ) in place of $\hat{\mathbf{C}} \backslash\{0\}$ (resp. $R$ ) since $\hat{\mathbf{C}} \backslash\{0\}$ (resp. $R$ ) does not admit the Green function. Let $g_{0}$ be the Green function on $\mathbf{D}$ with pole at 0 .

Definition 2.1 (cf. [B], $[\mathrm{BH}]$ ). We say that a subset $E$ of $\mathbf{D}_{0}$ is thin at 0 if ${ }^{\mathbf{D}} \hat{\mathbf{R}}_{g_{0}}^{E} \neq g_{0}$, where ${ }^{\mathbf{D}} \hat{\mathbf{R}}_{g_{0}}^{E}$ is the balayage of $g_{0}$ relative to $E$ on $\mathbf{D}$.

If $E$ is a closed subset of $\mathbf{D}$, it is well-known that $E$ is thin at 0 if and only if 0 is an irregular boundary point of $\mathbf{D} \backslash E$ in the sense of the Dirichlet problem.

The following lemma gives the quasiconformal invariance for thinness.

Lemma 2.1 (cf. [M], [Sh]). Let $G$ be a subdomain of $\mathbf{C}$ and $\varphi$ a quasiconformal mapping from $\mathbf{C}$ onto $\mathbf{C}$. If $\zeta$ is an irregular boundary point of $G$ in the sense of Dirichlet problem, $\varphi(\zeta)$ is an irregular boundary point of $\varphi(G)$ in the sense of Dirichlet problem.

Definition 2.2. A subset $U$ in $\mathbf{D}$ which contains 0 is said to be a fine neighborhood of 0 if $\mathbf{D} \backslash U$ is thin at 0 .

Let $k_{\zeta}$ be the Martin function on $R_{0}$ with pole at $\zeta \in \Delta^{R}$.

Definition 2.3. Let $\zeta$ be a point in $\Delta_{1}^{R}$ and $E$ a subset of $R_{0}$. We say that $E$ is minimally thin at $\zeta$ if ${ }^{R_{0}} \hat{\mathbf{R}}_{k_{\zeta}}^{E} \neq k_{\zeta}$.

DEFinition 2.4. Let $\zeta$ be a point in $\Delta_{1}^{R}$ and $U$ a subset of $R_{0}$. We say that $U \cup\{\zeta\}$ is a minimal fine neighborhood of $\zeta$ if $R_{0} \backslash U$ is minimally thin at $\zeta$.

The following proposition gives the characterization of $\# \Delta_{1}^{R}$ in terms of minimal fine topology.

Proposition 2.1 ([MS]). Let $\mathscr{M}$ be the class of subdomains $M$ of $\mathbf{D}_{0}$ such that $M \cup\{0\}$ is a fine neighborhood of $z=0$. Then, it holds that

$$
\# \Delta_{1}^{R}=\max _{M \in \mathscr{M}} n_{R}(M),
$$

where $n_{R}(M)$ is the number of connected components of $\pi^{-1}(M)$ and $\pi$ is the projection map from $R$ onto $\hat{\mathbf{C}} \backslash\{0\}$.

\section{§3. Proof of Main Theorem in case $p=2$}

Consider the case $p=2$ in this section. Let $R$ and $R^{\prime}$ be as in Main Theorem and $f$ be a quasiconformal mapping from $R$ onto $R^{\prime}$. It is known that $1 \leq \# \Delta_{1}^{R}, \# \Delta_{1}^{R^{\prime}} \leq 2$ (cf. [H] and see also [MS]). We have only to prove that $\# \Delta_{1}^{R^{\prime}}=2$ if and only if $\# \Delta_{1}^{R}=2$. Since $f^{-1}$ is a quasiconformal mapping from $R^{\prime}$ onto $R$, it is sufficient to prove that if $\# \Delta_{1}^{R}=2$, then $\# \Delta_{1}^{R^{\prime}}=2$. Suppose that $\# \Delta_{1}^{R}=2$. Let $\pi$ (resp. $\pi^{\prime}$ ) be the projection map from $R$ (resp. 
$R^{\prime}$ ) onto $\hat{\mathbf{C}} \backslash\{0\}$. By Proposition 2.1 there exists a subdomain $U$ of $\mathbf{D}_{0}$ such that $\mathbf{D}_{0} \backslash U$ is thin at $0, n_{R}(U)=2$ and $f\left(\pi^{-1}(U)\right) \subset R_{0}^{\prime}\left(:=\left(\pi^{\prime}\right)^{-1}\left(\mathbf{D}_{0}\right)\right)$. Let $U_{j}(j=1,2)$ be components of $\pi^{-1}(U)$. Since $R$ is a 2 -sheeted unlimited covering surface of $\hat{\mathbf{C}} \backslash\{0\}$, it is easily seen that each $U_{j}$ is considered as a replica of $U$. Let $g_{z}^{f\left(U_{j}\right)}(j=1,2)$ be the Green function on $f\left(U_{j}\right)$ with pole at $z$. Denote by $\mu_{f, j}$ the complex dilatation of $f$ on $U_{j}$. Set

$$
\mu_{j}= \begin{cases}\mu_{f, j} \circ \varphi_{j} & \text { on } U \\ 0 & \text { on } \mathbf{C} \backslash U,\end{cases}
$$

where $\varphi_{j}$ is the inverse of $\pi \mid U_{j}: U_{j} \rightarrow U$. It is well-known that there exists a quasiconformal mapping $f_{j}$ from $\mathbf{C}$ onto $\mathbf{C}$ with the complex delatation $\mu_{j}$ (cf. e.g. [LV]). Set $V_{j}=f_{j}(U)$. By Lemma 2.1 we find that $f_{j}(0)$ is an irregular boundary point of $V_{j}$ in the sense of the usual Dirichlet problem since 0 is an irregular boundary point of $U$ in the sense of the usual Dirichlet problem. On the other hand, the function $z \mapsto g_{f \circ \varphi_{j} \circ\left(f_{j}\right)^{-1}(z)}^{f\left(U_{U}\right)} \circ f \circ \varphi_{j} \circ\left(f_{j}\right)^{-1}(\xi)\left(\xi \in V_{j}\right)$ is the Green function on $V_{j}$ with pole at $\xi$ since $f \circ \varphi_{j} \circ\left(f_{j}\right)^{-1}$ is conformal. Hence, by [H1, Theorem 10.16], there exists a fine limit $\mathscr{F}-\lim _{z \rightarrow f_{j}(0)} g_{f \circ \varphi_{j} \circ\left(f_{j}\right)^{-1}(z)}^{f\left(U_{j}\right)} \circ f \circ \varphi_{j} \circ$ $\left(f_{j}\right)^{-1}$. Since $f_{j}(0)$ is an irregular boundary point of $V_{j}$ in the sense of the usual Dirichlet problem, this limit must be positive by [Hl, Theorem 8.34]. Denote this limit function on $V_{j}$ by $g_{0}^{V_{j}}$ and set $g_{0}^{f\left(U_{j}\right)}=g_{0}^{V_{j}} \circ f_{j} \circ \pi \circ f^{-1}$. We see that each $g_{0}^{f\left(U_{j}\right)}$ is a positive harmonic function on $f\left(U_{j}\right)$ since each $g_{0}^{V_{j}}$ is a positive harmonic function on $V_{j}$ and $f_{j} \circ \pi \circ f^{-1}$ is conformal. For $j=1,2$, set

$$
S_{j}\left(g_{0}^{f\left(U_{j}\right)}\right)(x):=\inf _{s} s(x),
$$

where $s$ runs over the space of positive superharmonic functions $s$ on $R_{0}^{\prime}$ satisfying $s \geq g_{0}^{f\left(U_{j}\right)}$ on $f\left(U_{j}\right)$. By Perron-Wiener-Brelot method we find that each $S_{j}\left(g_{0}^{f\left(U_{j}\right)}\right)$ is a positive harmonic function on $R_{0}^{\prime}$. Then, the following inequality

$$
S_{j}\left(g_{0}^{f\left(U_{j}\right)}\right)-R_{0}^{\prime} \hat{\mathbf{R}}_{S_{j}\left(g_{0}^{f\left(U_{j}\right)}\right)}^{R^{\prime} \backslash f\left(U_{j}\right)} \geq g_{0}^{f\left(U_{j}\right)}
$$

holds on $f\left(U_{j}\right)$. In fact, to prove the inequality $(*)$ note that

$$
R_{0}^{\prime} \hat{\mathbf{R}}_{S_{j}\left(g_{0}^{f\left(U_{j}\right)}\right)}^{R_{\prime}^{\prime} \backslash f\left(U_{j}\right)}=H_{S_{j}\left(g_{0}^{f\left(U_{j}\right)}\right)}^{f\left(U_{j}\right)}
$$

on $f\left(U_{j}\right)$, where $H_{S_{j}\left(g_{0}^{f\left(U_{j}\right)}\right)}^{f\left(U_{j}\right)}$ is the Dirichlet solution for $S_{j}\left(g_{0}^{f\left(U_{j}\right)}\right)$ on $f\left(U_{j}\right)$ (cf. e.g. [Hl], [CC]). By definition $S_{j}\left(g_{0}^{f\left(U_{j}\right)}\right) \geq g_{0}^{f\left(U_{j}\right)}$ on $f\left(U_{j}\right)$. Hence, by the definition of the Dirichlet solution in the sense of Perron-Wiener-Brelot,

on $f\left(U_{j}\right)$. Thus $(*)$ is proved.

$$
S_{j}\left(g_{0}^{f\left(U_{j}\right)}\right)-g_{0}^{f\left(U_{j}\right)} \geq H_{S_{j}\left(g_{0}^{f\left(U_{j}\right)}\right)}^{f\left(U_{j}\right)}
$$

We shall proceed the proof of Main Theorem in case $p=2$. By the Martin representation theorem, there exist at most two minimal functions $h_{j, k}(k=1,2)$ on $R_{0}^{\prime}$ with $S_{j}\left(g_{0}^{f\left(U_{j}\right)}\right)=h_{j, 1}+h_{j, 2}$ on $R_{0}^{\prime}$. Hence, by the above inequality $(*)$, we have 


$$
h_{j, 1}+h_{j, 2}=S_{j}\left(g_{0}^{f\left(U_{j}\right)}\right) \geq{ }^{R_{0}^{\prime}} \hat{\mathbf{R}}_{h_{j, 1}+h_{j, 2}}^{R_{j}^{\prime} \backslash f\left(U_{j}\right)}+g_{0}^{f\left(U_{j}\right)}>{ }^{R_{0}^{\prime}} \hat{\mathbf{R}}_{h_{j, 1}}^{R_{0}^{\prime} \backslash f\left(U_{j}\right)}+{ }^{R_{0}^{\prime}} \hat{\mathbf{R}}_{h_{j, 2}}^{R_{0}^{\prime} \backslash f\left(U_{j}\right)}
$$

on $f\left(U_{j}\right)$. Therefore, we find that there exists a minimal function $h_{j}$ on $R_{0}^{\prime}$ such that $h_{j} \neq R_{0}^{\prime} \hat{\mathbf{R}}_{h_{j}}^{R_{0}^{\prime} \backslash f\left(U_{j}\right)}$. Hence, by the definition of minimal thinness, $R_{0}^{\prime} \backslash f\left(U_{j}\right)$ is minimally thin at the minimal boundary point corresponding to $h_{j}$. Since $f\left(U_{1}\right) \cap f\left(U_{2}\right)=\emptyset$, we find that $\# \Delta_{1}^{R^{\prime}}=2$.

\section{§4. Proof of Main Theorem in case $p=3$}

Consider the case $p=3$ in this section. As in $\S 3$, it is known that $1 \leq \# \Delta_{1}^{R}$, $\# \Delta_{1}^{R^{\prime}} \leq 3$ (cf. [H] and see also [MS]). By the same argument as in the proof of Main Theorem in case $p=2$ we find that $\# \Delta_{1}^{R}=3$ if and only if $\# \Delta_{1}^{R^{\prime}}=3$. Hence, to prove the statement of Main Theorem in case $p=3$, we have only to prove that $\# \Delta_{1}^{R^{\prime}}=2$ if and only if $\# \Delta_{1}^{R}=2$. Since $f^{-1}$ is a quasiconformal mapping from $R^{\prime}$ onto $R$, it is sufficient to prove that if $\# \Delta_{1}^{R}=2$, then $\# \Delta_{1}^{R^{\prime}}=2$. Contrary to this, we suppose that $\# \Delta_{1}^{R}=2$ and that $\# \Delta_{1}^{R^{\prime}} \neq 2$. Then, by the above observation, we see that $\# \Delta_{1}^{R^{\prime}}=1$.

By Proposition 2.1 there exists a subdomain $U$ of $\mathbf{D}_{0}$ such that $\mathbf{D}_{0} \backslash U$ is thin at $0, n_{R}(U)=2$ and $f\left(\pi^{-1}(U)\right) \subset R_{0}^{\prime}$. Hence $\pi^{-1}(U)$ consists of two connected components $U_{1}$ and $U_{2}$. Since $R$ is a 3 -sheeted unlimited covering surface of $\hat{\mathbf{C}} \backslash\{0\}$, we assume that $U_{1}$ is a 1 -sheeted unlimited covering surface of $U$, that is, $U_{1}$ is a replica of $U$ and $U_{2}$ is a 2-sheeted unlimited covering surface of $U$. Let $g_{z}^{f\left(U_{1}\right)}$ be the Green function on $f\left(U_{1}\right)$ with pole at $z$. Denote by $\mu_{f}$ the complex dilatation of $f$ on $U_{1}$. Set

$$
\mu= \begin{cases}\mu_{f} \circ \varphi & \text { on } U \\ 0 & \text { on } \mathbf{C} \backslash U,\end{cases}
$$

where $\varphi$ is the inverse of $\pi \mid U_{1}: U_{1} \rightarrow U$. It is well-known that there exists a quasiconformal mapping $\psi$ from $\mathbf{C}$ onto $\mathbf{C}$ with the complex delatation $\mu$ (cf. [LV]). By the same method as in $\S 3$, there exists a positive fine limit $\mathscr{F}-\lim _{z \rightarrow \psi(0)} g_{z}^{f\left(U_{1}\right)} \circ f \circ \varphi \circ \psi^{-1}$. Denote by $g_{0}^{f\left(U_{1}\right)}$ the pull-back of this limit function on $\psi\left(U_{1}\right)$ by $\psi \circ \pi \circ f^{-1}$. We see that $g_{0}^{f\left(U_{1}\right)}$ is a positive harmonic function on $f\left(U_{1}\right)$. Set

$$
S\left(g_{0}^{f\left(U_{1}\right)}\right)(x):=\inf _{s} s(x)
$$

where $s$ runs over the space of positive superharmonic functions $s$ on $R_{0}^{\prime}$ satisfying $s \geq g_{0}^{f\left(U_{1}\right)}$ on $f\left(U_{1}\right)$. By Perron-Wiener-Brelot method we find that $S\left(g_{0}^{f\left(U_{1}\right)}\right)$ is a positive harmonic function on $R_{0}^{\prime}$. By the same consideration as in the proof of Main Theorem in case $p=2$, we have

$$
S\left(g_{0}^{f\left(U_{1}\right)}\right)-{ }^{R_{0}^{\prime}} \hat{\mathbf{R}}_{S\left(g_{0}^{f\left(U_{1}\right)}\right)}^{R^{\prime} \backslash f\left(U_{1}\right)} \geq g_{0}^{f\left(U_{1}\right)}
$$

on $f\left(U_{1}\right)$. By the assumption, $S\left(g_{0}^{f\left(U_{1}\right)}\right)$ is only one minimal harmonic function on $R_{0}^{\prime}$. Hence, by $(* *)$, we find that $R_{0}^{\prime} \backslash f\left(U_{1}\right)$ is minimally thin at the minimal 
boundary point corresponding to $S\left(g_{0}^{f\left(U_{1}\right)}\right)$. Take a curve $\gamma$ in $U$ such that $\gamma$ reaches 0 and that $\pi^{-1}(\gamma)$ does not possess any branch points of $R$. Hence there exists a curve $\tilde{\gamma}$ in $U_{2}\left(\subset R_{0} \backslash U_{1}\right)$ with $f(\tilde{\gamma}) \subset R_{0}^{\prime}$ and $\pi(\tilde{\gamma})=\gamma$ which reaches the ideal boundary since $R$ is unlimited. Hence this implies that

i) $f(\tilde{\gamma})$ is a subset of $R_{0}^{\prime} \backslash f\left(U_{1}\right)$;

ii) $\pi^{\prime}(f(\tilde{\gamma}))$ is not thin at 0 in the usual sense, where $\pi^{\prime}$ is the projection map from $R^{\prime}$ onto $\hat{\mathbf{C}} \backslash\{0\}$.

By the above fact i), $f(\tilde{\gamma})$ is minimally thin at the minimal boundary point corresponding to $S\left(g_{0}^{f\left(U_{1}\right)}\right)$. On the other hand, by the above fact ii) and [MS, Proposition 3.1], $f(\tilde{\gamma})$ is not minimally thin at the minimal boundary point corresponding to $S\left(g_{0}^{f\left(U_{1}\right)}\right)$. This is a contradiction. We have the desired result.

\title{
REFERENCES
}

[AS] L. V. Ahlfors and L. SARio, Riemann Surfaces, Princeton, 1960.

[B] M. BRelot, On Topologies and Boundaries in Potential Theory, Lecture Notes in Math. 175, Springer, 1971.

[BH] J. Bliedtner and W. Hansen, Potential Theory, Springer, 1986.

[CC] C. Constantinescu and A. CoRnea, Ideale Ränder Riemanncher Flächen, Springer, 1969.

[F] O. Forster, Lectures on Riemann Surfaces, GTM 81, Springer.

[H] M. HeIns, Riemann surfaces of infinite genus, Ann. of Math. 55 (1952), 296-317.

[Hl] L. Helms, Introduction to Potential Theory, Wiley-Interscience, 1969.

[L] T. LyONS, Instability of the Liouville property for quasi-isometric Riemannian manifolds and reversible Markov chains, J. Differential Geometry 26 (1987), 33-66.

[LV] O. Lehto AND K. I. Virtanen, Quasiconformal Mappings in the Plane, Springer, 1973.

[M] H. Masaoka, Quasiregular mappings and $d$-thinness, Osaka J. Math. 34 (1997), 223-231.

[MS] H. Masaoka and S. Segawa, Harmonic dimension of covering surfaces and minimal fine neighborhood, Osaka J. Math. 34 (1997), 659-672.

[SN] L. Sario AND M. NAKaI, Classification Theory of Riemann Surfaces, Springer, 1970.

[Sh] H. SHIGA, Quasiconformal mappings and potentials, XVIth Rolf Nevanlinna Colloquium, Walter de Gruyter \& Co., 1996, 215-222.

\author{
HIROAKI MASAOKA \\ Department of Mathematics \\ FACUlty OF SCIENCE \\ Kyoto SANGyo University \\ Kamigamo-Motoyama, KitaKU \\ Куото 603-8555 \\ JAPAN \\ e-mail: masaoka@cc.kyoto-su.ac.jp \\ Shigeo Segawa \\ Department of Mathematics \\ Daido Institute of TeChNology \\ NAGOYA $457-8530$ \\ JAPAN \\ e-mail: segawa@daido-it.ac.jp
}

